\title{
A new prognostic scale for the early prediction of ischemic stroke recovery mainly based on traditional Chinese medicine symptoms and NIHSS score: a retrospective cohort study
}

\author{
Ke-Gang $\mathrm{Cao}^{1 \dagger}$, Cai-Hong $\mathrm{Fu}^{1+}{ }^{1+}$, Huan-Qin $\mathrm{Li}^{2}, \mathrm{Xi}^{-}$Yan $\mathrm{Xin}^{3}$ and Ying Gao ${ }^{1 *}$
}

\begin{abstract}
Background: Ischemic stroke (IS) is a common disease, often resulting in death or disability. Previous studies on prognosis of stroke mainly focused on the baseline condition or modern expensive tests. However, the change of clinical symptoms during acute stage is considerably neglected. In our study, we aim to develop a new prognostic scale to predict the 90-day outcome of IS patients.

Methods: In this retrospective cohort study, a secondary data analysis was performed on 489 patients extracted from 1046 patients of 4 hospitals. A new prognostic scale was constructed to predict the recovery of IS mainly based on the National Institutes of Health Stroke Scale (NIHSS) score, traditional Chinese Medicine (TCM) symptoms \& signs and the changes during the first 3 days of patients in the 3 TCM hospitals. Receiver Operating Characteristic (ROC) curve was used to determine the cutoff point for prediction. In the end, the scale was used to test the outcome of IS patients in Xuanwu hospital.

Results: The new prognostic scale was composed of 8 items including age degree (OR=3.32; $95 \% \mathrm{Cl}$ : 1.72-6.42), history of diabetes mellitus (DM) $(\mathrm{OR}=2.20 ; 95 \% \mathrm{Cl}: 1.19-4.08)$, NIHSS score $(\mathrm{OR}=3.08$; $95 \% \mathrm{Cl}: 2.16-4.40)$, anxiety $(\mathrm{OR}=3.17 ; 95 \% \mathrm{Cl}: 1.90-5.29)$ and irritability $(\mathrm{OR}=4.61 ; 95 \% \mathrm{Cl}: 1.36-15.63)$ on the 1st day of illness onset, change in NIHSS score (OR=2.49; $95 \% \mathrm{Cl}: 1.31-4.73)$, and circumrotating (OR=7.80; $95 \% \mathrm{Cl}: 1.98-30.64)$ and tinnitus $(\mathrm{OR}=13.25 ; 95 \% \mathrm{Cl}: 1.55-113.34)$ during the first 3 days of stroke onset. The total score of the scale was 16.5 and the cutoff point was 9.5, which means patients would have poor outcome at 90 days of stroke onset if the score was higher than 9.5. The new scale was validated on the data of Xuanwu hospital, and the value of its sensitivity, specificity and overall accuracy were $69.6 \%, 83.3 \%$ and $75.0 \%$ respectively.

Conclusions: The 8-item scale, mainly based on TCM symptoms, NIHSS score and their changes during the first 3 days, can predict the 90-day outcome for IS patients while it still needs to be further validated and optimized clinically.
\end{abstract}

\footnotetext{
* Correspondence: gaoying973@126.com

${ }^{\dagger}$ Equal contributors

'Department of Neurology, Dongzhimen Hospital affiliated to Beijing University of Chinese Medicine, Beijing, China

Full list of author information is available at the end of the article
} 


\section{Background}

Ischemic stroke (IS) is a common disease with the characteristics of high incidence, high mortality, severe morbidity, high recurrence rate and serious complications, and it imposes a huge economic burden on the family and the society [1-7]. Stroke has been listed as the third leading cause of death following heart diseases and cancer in the world $[3,4,6]$. Ischemic stroke accounts for approximately $80 \%$ of the total cerebrovascular diseases [8]. Therefore, the prevention and treatment of ischemic stroke has attracted a great attention from the medical profession domestically and internationally in recent years $[9,10]$. Meanwhile the judgment of stroke prognosis plays an important role in the formulation of health and economic policy and the choice of treatment programs.

Prognosis, affecting both initial therapy and rehabilitation plans of ischemic stroke patients, has been studied for several decades [11]. Previous studies on prognosis of ischemic stroke was mainly focused on the baseline condition or some modern expensive tests like Magnetic Resonance diffusion-weighted Imaging (MR DWI) [12], as well as static signs, such as obviously unconscious [13]. Recently, some researchers have attached importance to the change of signs, such as blood pressure (BP) during the acute stage [14]. However, the change of clinical symptoms during acute stage is consistently neglected. A comprehensive and precise prognosis scale of ischemic stroke still needs to be further developed [15].

The National Institutes of Health Stroke Scale (NIHSS) is widely used to evaluate the severity of neurological deficit of stroke patients [16, 17]. Barthel Index (BI) is the most commonly used tool to evaluate the physical disability and functional recovery after stroke $[18,19]$. Modern medical studies also show that these factors such as age, gender, hypertension, diabetes, smoking, and drinking alcohol, have a close relationship with the prognosis of stroke [6,20,21].

In Traditional Chinese Medicine (TCM), symptoms or syndromes can influence the diagnosis and treatment of the disease greatly. Many researchers analyse and induce TCM Symptoms or syndrome by means of modern algorithms to be convenient in clinical application [22-24]. Several early symptoms such as anxiety, irritability and circumrotating related to stroke outcomes were not only mentioned in ancient literature [25], but also reported by TCM doctors nowadays [26, 27]. It is important for clinical doctors to decide the treatment plan based on these symptoms \& signs within the first few hours after stroke onset and predict the likely outcome of patients. Knowing the prognosis of stroke is also very important for stroke patients and their families [12, 28].

However, TCM symptoms have not been applied as the early predictive indicators of stroke outcome in the prognostic scale of stroke. Based on NIHSS score, TCM symptoms and their changes during the first 3 days of stroke onset, we developed a new scale to predict the 90-day outcome in the current study.

\section{Methods}

\section{Study design}

In the retrospective cohort study, a secondary data analysis was performed on a Microsoft ACCESS database among ischemic stroke patients based on the project of Major State Basic Research Development Program of China (973 Program NO.2003CB517102) (Fig. 1).

\section{Ethics}

The study protocol was conformed to the Helsinki Declaration [29] and the research regulations for Chinese clinical trials, which was approved by the Ethics Committee of Dongzhimen Hospital affiliated to Beijing University of Chinese Medicine.

\section{Study subjects}

1046 cases of ischemic stroke patients were recruited between January, 2004 to December, 2009 from inpatient, outpatient, and emergency department settings in 4 hospitals including three TCM hospitals and one western medicine hospital: Dongzhimen Hospital affiliated to Beijing University of Chinese Medicine, Second Affiliated Hospital of Tianjin University of TCM, Guangdong Provincial Chinese Medicine Hospital and Xuanwu Hospital affiliated to Capital Medical University. All ischemic stroke patients received the standard treatment according to World Health Organization (WHO) criteria [30].

\section{Diagnostic criteria}

(1) Western Medicine diagnostic criteria of ischemic stroke referred to WHO criteria and intracranial hemorrhage was further excluded by Computed Tomography $(\mathrm{CT})$ or Magnetic Resonance Imaging (MRI) scan in the hospital [30]. (2) TCM diagnostic criteria with reference to the "Standards of TCM Syndrome-differentiated Diagnosis of Apoplexy" were regulated by the Encephalopathy Accident and Emergency Collaborative Group of State Administration of Traditional Chinese Medicine in 1995 [31].

\section{Inclusion criteria}

(1) All patients should conform to the diagnostic criteria of ischemic stroke in Western medicine and apoplexy; (2) Ischemic stroke can be definitely diagnosed by CT or MRI scan; (3) Patients arriving in hospital within $24 \mathrm{~h}$ of stroke onset were included. (4) NIHSS scores, TCM symptoms \& signs of the first 3 days after stroke onset and data of BI on 90th day were completely recorded. 


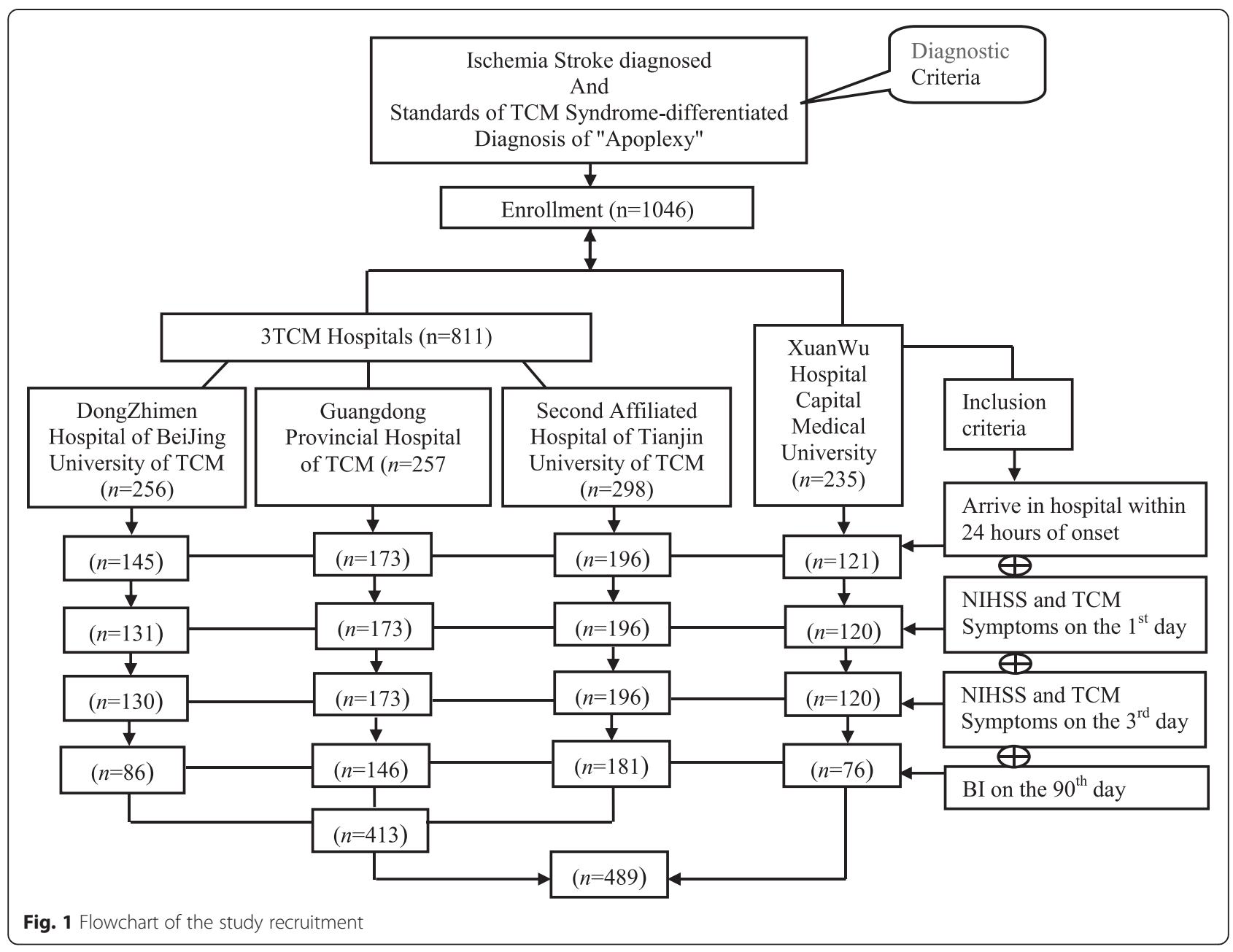

\section{Exclusion criteria}

The exclusion criteria were including: (1) patients with cerebral transient ischemic attacks (TIA); (2) cerebral apoplexy caused by blood diseases, cancer or other causes; (3) accompanying some serious illnesses, such as gastrointestinal hemorrhage, heart or kidney failure, cancer, osteoarthritis and so on; (4) patients with mental disorder or severe dementia; (5) patients with obvious sequel of apoplexy.

\section{Data collection}

The observed data including patients' demographics (age, gender, medical history, etc.), auxiliary examination, stroke onset time and admission time, and TCM clinical symptoms \& signs were recorded on the case report form (CRF) by neurologists. TCM symptoms \& signs were recorded for the 1st, 2nd, 3rd day and 90th day after stroke onset. The NIHSS was performed by researchers with NIHSS certificate on the 1st, 3rd, 7th, 14th, 28th, and 90th day in the hospital, and the score were recorded. BI on the 90th day of patients was also recorded. For the accuracy and consistence of the data in collecting and testing, a monitoring system for the collection of information and standardized abstraction forms was applied to guide the data collection in case of ambiguous, conflicting, and missing information.

\section{Measurements}

\section{Outcome measurement}

The BI as a reliable and valid assessment scale can be used to assess the function of ten activities in daily life $[32,33]$. The maximum score of 100 indicates a full independence. Good stroke recovery was defined as BI score of 90 or higher, indicating a near full functional independence on the 90th day $[34,35]$.

\section{Predictors}

The NIHSS is widely used to measure the neurological function and predict the outcome of stroke in the clinical practice. It consists eleven graded items including level of consciousness, language function, visual fields, eye movements, facial symmetry, motor strength, sensation, coordination and so on, which has undergone extensive validation and reliable assessments [36]. Clinical 
nerve function defect could change dramatically during the first 48 to $72 \mathrm{~h}$ after stroke onset [37]. In this study, the change in NIHSS scores would be recorded from the difference of scores between the 3rd day and the 1st day of stroke onset [14].

TCM symptoms \& signs with appearance rate $\geq 5 \%$ were selected from the database in the actual data analysis. The changes in TCM symptoms \& signs during the first 3 days were expressed as disappearance of symptom (disappear), no change (keep) and new appearance (appear) respectively.

In addition, the demographic and clinical factors were assessed, such as age, sex, history of stroke, hypertension, coronary heart disease (CHD), atrial fibrillation (AF), and diabetes mellitus (DM), which were defined as patients with medical treatment history.

\section{Statistical analysis}

The data were analyzed by SPSS statistical software version 12.0 (SPSS, Chicago, USA). The measurement data such as age, NIHSS score, BI score and course of disease were expressed as mean \pm standard deviation and the enumeration data such as gender and medical history were expressed as counts (percentage). Frequency comparisons were made with the method of Chi-square test. Two-group comparisons of normally distributed data were performed with the independent samples $t$-test. Mann-Whitney $U$ test was used to analyze the difference of two groups for these unmatched normally distributed data. Correlation between the TCM symptoms \& signs and NIHSS score was analyzed by Spearman ranked correlation coefficient. The method of logistic regression was used to further screen prognostic factors and recursive partitioning and classification tree for optimum discrimination of variables classification between good and poor recovery. The cutoff point for the prognostic model of ischemic stroke recovery was determined by Receiver Operating Characteristic (ROC) curve with maximum Youden's index value. A probability of $P<0.05$ was considered to be statistically significant.

\section{Results}

\section{Participants}

From the total 1046 subjects in the database, 489 ischemic stroke patients were finally enrolled into the study according to the diagnostic criteria and inclusive criteria, which included 413 cases from 3 TCM hospitals and 76 cases from Xuanwu hospital (Fig. 1).

\section{Baseline characteristics of the patients}

Table 1 showed the demographics and the baseline characteristics of the 489 patients based on the outcome details with good recovery (good recovery was defined as a score of 90 or higher on the 90 -day) $(n=278)$ and poor recovery $(n=211)$ measured by BI. The average age of all the patients was 65.8 years old and $63.0 \%(n=308)$ were men. The average score of NIHSS and BI of all the stroke patients were 6.2 and 77.9 respectively. There were no statistical differences $(P>0.05)$ between the 3 TCM hospitals and Xuanwu hospital in these demographics and the baseline characteristics including the history of hypertension, stroke, CHD, DM, AF, course of disease, NIHSS scores, and BI score. And the data of these hospitals were comparable.

Table 1 The demographic and baseline characteristics of patients

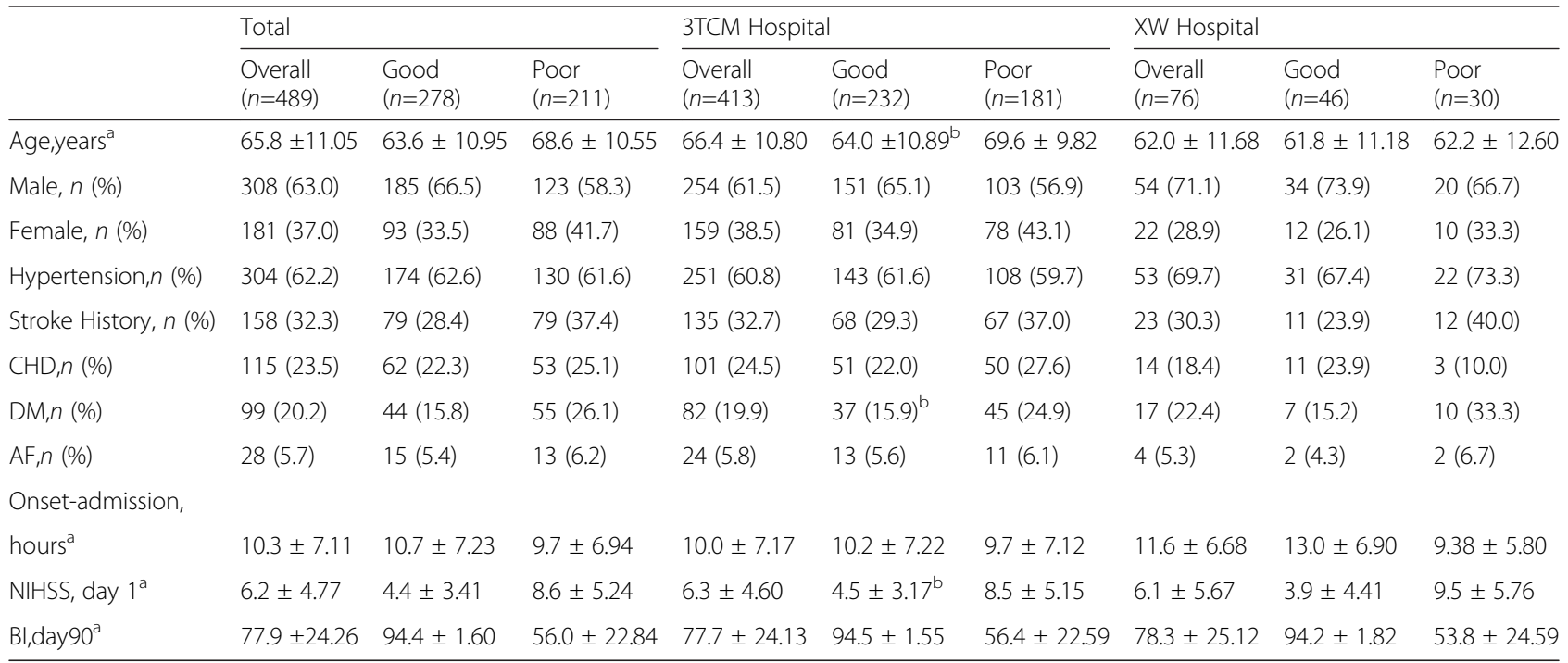

TCM traditional Chinese medicine, XW Xuanwu, CHD coronary heart disease, DM Diabetes Mellitus, AF Atrial Fibrillation, NIHSS National Institutes of Health Stroke Scale, BI Barthel Index. Good good recovery, Poor bad recovery

${ }^{\mathrm{a}}$ Data presented as mean \pm SD. ${ }^{\mathrm{b}}$ There were statistical differences $(P<0.05)$ between the good and poor recovery 
Table 2 The relationship between TCM symptoms \& signs and NIHSS score

\begin{tabular}{lcc}
\hline TCM symptom \& signs & Correlation coefficient $\left(r^{2}\right)$ & $P$ value \\
\hline Limb flaccid paralysis & 0.451 & 0.000 \\
Incontinence of urinary & 0.325 & 0.000 \\
Cough due to drinking & 0.308 & 0.000 \\
Respiratory rude & 0.272 & 0.000 \\
Irritability & 0.255 & 0.000 \\
Anxiety & 0.249 & 0.000 \\
Analeptic & 0.186 & 0.000 \\
Stiffness and tension in the limbs & 0.132 & 0.003 \\
Purple lips & 0.108 & 0.017 \\
Salivation & 0.090 & 0.047 \\
Tinnitus & -0.099 & 0.028 \\
Thirst with desire to hot drink & -0.104 & 0.022 \\
Frequent micturition & -0.107 & 0.018 \\
Dry mouth & -0.117 & 0.009 \\
Circumrotating & -0.141 & 0.002 \\
Dizziness & -0.193 & 0.000 \\
\hline
\end{tabular}

TCM traditional Chinese medicine. Significant difference, $P<0.05$

\section{The relationship between TCM symptoms and NIHSS} score

In practical analysis, 57 TCM symptoms [see Additional file 1: Table S1] were selected with the appearance rate $\geq$ $5 \%$ from 157 TCM symptoms \& signs except tongue and pulse. Then, the relationship between these TCM symptoms \& signs and NIHSS score on the 1st day of stroke onset were analysed with Pearson Correlation, and the results demonstrated that 16 of them were related to NIHSS score closely $(P<0.05)$. Moreover, the highest correlation coefficient $\left(\mathrm{r}^{2}\right)$ was 0.451 (Table 2).

\section{Construction of the new scale Screening of the prognostic factors}

The new scale was developed based on the data of 3 TCM hospitals. From the general information of patients on the 1st day on admission, age, history of DM and NIHSS score were screened out as the significant prognostic indicators of ischemic stroke (Table 1).

Table 3 showed there were statistical differences $(P<$ $0.05)$ in 14 TCM symptoms \& signs on the 1st day, which were screened from 57 TCM symptoms \& signs, between the good and poor recovery measured by BI from the data of 3 TCM hospitals. And the changes of NIHSS score, irritability, circumrotating, tinnitus and difficult to cough sputum during the first 3 days were also related to the outcome (Tables 3 and 4).

Among the selecting factors above, the age, NIHSS score on the 1st day and the change of NIHSS score during the first 3 days were dealt with as continuous variables for optimum discrimination between good and poor recovery by the method of classification tree [12]. The analysis results showed that the two categories of age degree were assigned $\leq 56.44$ or $>56.44$, the three categories of NIHSS score (on the 1st day) $\leq 2,3-6$, or $>$ 7 , the two categories of change in NIHSS score (during the first 3 days) $\leq-1$, or $>-1$ (Fig. 2).

Table 3 Comparison of TCM symptoms \& signs between good and poor recovery for 3 TCM hospitals

\begin{tabular}{|c|c|c|c|}
\hline Factors & Good $(n=232)$ & Poor $(n=181)$ & $P$ value \\
\hline Spiritlessness, day 1,n (\%) & $107(46.1)$ & $105(58.0)$ & 0.016 \\
\hline Anxiety day $1, n(\%)$ & $49(21.1)$ & $94(51.9)$ & 0.000 \\
\hline Cough due to drinking, day $1, n(\%)$ & $48(20.7)$ & $69(38.1)$ & 0.000 \\
\hline Anorexia,day $1, n(\%)$ & $27(11.6)$ & $39(21.5)$ & 0.006 \\
\hline Salivation, day $1, n(\%)$ & $22(9.5)$ & $31(17.1)$ & 0.021 \\
\hline Analeptic, day $1, n(\%)$ & $17(7.3)$ & $27(14.9)$ & 0.013 \\
\hline Limb flaccid paralysis, day $1, n(\%)$ & $11(4.7)$ & $28(15.5)$ & 0.000 \\
\hline Thirst with desire to hot drink, day $1, n(\%)$ & $25(10.8)$ & $9(5.0)$ & 0.033 \\
\hline Circumrotating, day $1, n(\%)$ & $25(10.8)$ & $8(4.4)$ & 0.018 \\
\hline Emaciation,n (\%) & $9(3.9)$ & $24(13.3)$ & 0.000 \\
\hline Stiffness and tension in the limbs, day $1, n(\%)$ & $12(5.2)$ & $22(12.2)$ & 0.010 \\
\hline Tinnitus, day $1, n(\%)$ & $23(9.9)$ & $5(2.8)$ & 0.004 \\
\hline Irritability, day 1,n (\%) & $5(2.2)$ & $18(9.9)$ & 0.001 \\
\hline Incontinence of urinary, day $1, n(\%)$ & $10(4.3)$ & 19 (10.5) & 0.015 \\
\hline
\end{tabular}

Significant difference, $P<0.05$

Good: good recovery; Poor: bad recovery

${ }^{*} P$ values based on Chi-square test 
Table 4 The changes of NIHSS and TCM symptoms between good and poor recovery for 3 TCM hospitals

\begin{tabular}{|c|c|c|c|c|}
\hline Factors & Total $(n=413)$ Change status & Good $(n=232)$ & Poor $(n=181)$ & $P$ value \\
\hline NIHSS & - & $0.54 \pm 1.80$ & $-0.45 \pm 2.74$ & $0.000^{a}$ \\
\hline \multicolumn{5}{|l|}{ TCM symptoms \& signs } \\
\hline \multirow[t]{3}{*}{ Irritability } & appear, $n(\%)$ & $0(0.0)$ & $3(1.7)$ & 0.037 \\
\hline & keep,n (\%) & $230(99.1)$ & $166(91.7)$ & \\
\hline & disappear,n (\%) & $2(0.9)$ & $12(6.6)$ & \\
\hline \multirow[t]{3}{*}{ Circumrotating } & appear,n (\%) & $0(0.0)$ & $4(2.2)$ & 0.007 \\
\hline & keep,n (\%) & $220(94.8)$ & $174(96.1)$ & \\
\hline & disappear,n (\%) & $12(5.2)$ & $3(1.7)$ & \\
\hline \multirow[t]{3}{*}{ Tinnitus } & appear,n (\%) & $1(0.4)$ & $2(1.1)$ & 0.002 \\
\hline & keep,n (\%) & $219(94.4)$ & $179(98.9)$ & \\
\hline & disappear,n (\%) & $12(5.2)$ & $0(0.0)$ & \\
\hline \multirow[t]{3}{*}{ Difficult to cough sputum } & appear,n (\%) & $4(1.7)$ & $7(3.9)$ & 0.031 \\
\hline & keep,n (\%) & $221(95.3)$ & $173(95.6)$ & \\
\hline & disappear,n (\%) & $7(3.0)$ & $1(0.6)$ & \\
\hline
\end{tabular}

TCM traditional Chinese medicine. NIHSS National Institutes of Health Stroke Scale ${ }^{a}$ Mann-Whitney $U$ test; Good: good recovery; Poor: bad recovery

The method of logistic regression was used to further screen prognostic factors from all variables in Tables 1, 3 and 4 . On statistical analysis, there were 8 independent prognosis variables were included in the prognosis model: age [Odds ratio (OR) 3.32, $P<0.05$ ], history of DM (OR 2.20, $P<0.05$ ), anxiety (OR 3.17, $P<0.05$ ), NIHSS score (OR 3.08, $P<0.05$ ), irritability on the 1st day (OR 4.61, $P<0.05$ ), and the changes in NIHSS score (OR 2.49, $P<0.05$ ), circumrotating (OR 7.80, $P<0.05$ ) and tinnitus (OR 13.25, $P<0.05)$ during the first 3 days (Table 5).

\section{Adding weight value for each prognostic factors}

In order to use the prognostic model conveniently and effectively in clinic, different points were assigned for the categories of the 8 factors by a simple weighting scheme according to the value of findings and analysis. The results of categories obtained were as follows: age degree $[0(\leq 56.44) ; 1(>56.44)]$, history of DM [0 (no); 1 (yes)], anxiety on the 1st day [0 (no); 1 (yes)], NIHSS score degree on the 1st day $[0(\leq 2) ; 1$ $(3-6) ; 2(>7)]$, irritability on the 1 st day $[0$ (no); 1.5 (yes)], change in NIHSS score during the first 3 days

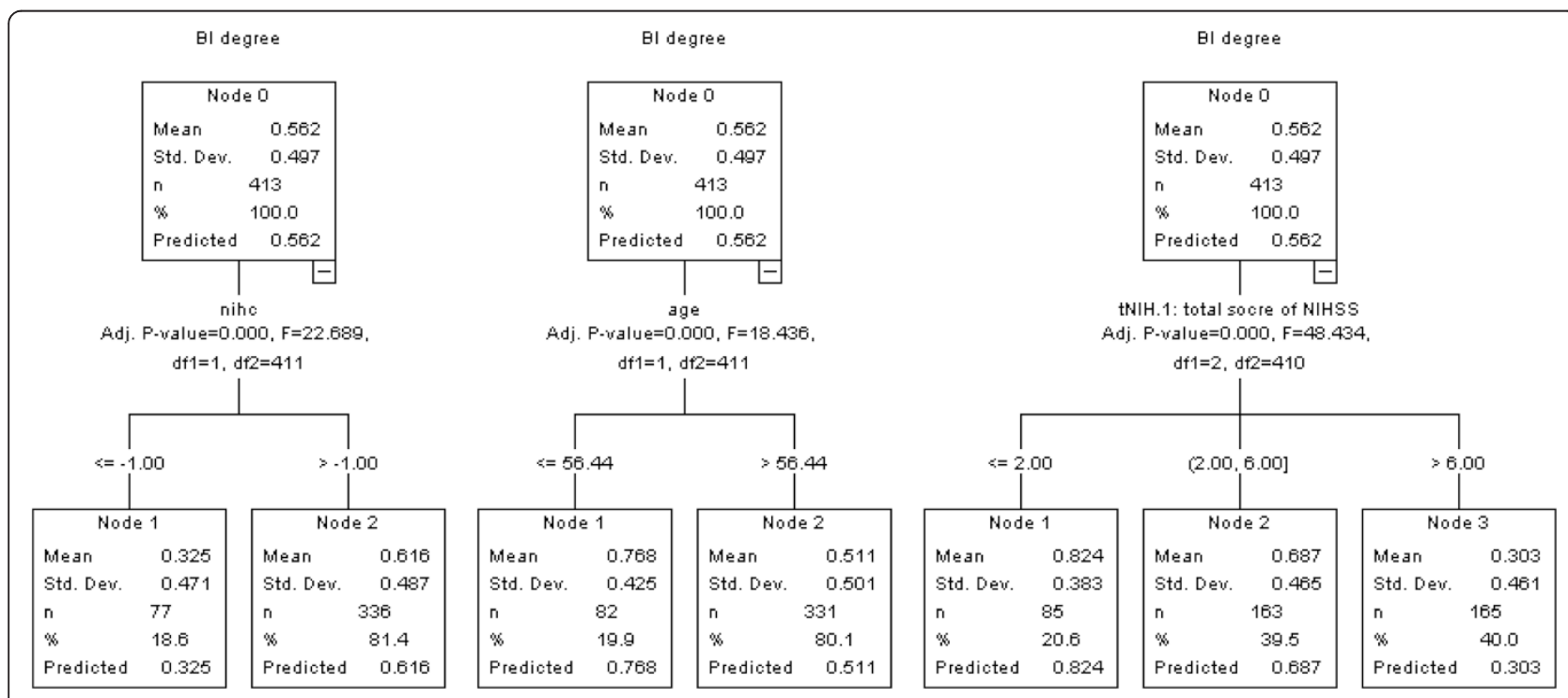

Fig. 2 The optimum discrimination of some independent prognosis variables by the method of Classification Tree 
Table 5 Screening prognostic fators by the method of logistical regression model in 3 TCM hospitals

\begin{tabular}{llll}
\hline Factor & Coefficient (S.E.) & OR (95 \% Cl) & $P$ value \\
\hline Age degree & $1.20(0.34)$ & $3.32(1.72-6.42)$ & 0.000 \\
History of DM & $0.79(0.32)$ & $2.20(1.19-4.08)$ & 0.012 \\
Anxiety, day 1 & $1.15(0.26)$ & $3.17(1.90-5.29)$ & 0.000 \\
NIHSS score degree, day 1 & $1.12(0.18)$ & $3.08(2.16-4.40)$ & 0.000 \\
Irritability, day 1 & $1.53(0.62)$ & $4.61(1.36-15.63)$ & 0.014 \\
Change in NIHSS score during 3 days & $0.91(0.33)$ & $2.49(1.31-4.73)$ & 0.006 \\
Change in circumrotating during 3 days & $2.05(0.70)$ & $7.80(1.98-30.64)$ & 0.003 \\
Change in tinnitus during 3 days & $2.58(1.10)$ & $13.25(1.55-113.34)$ & 0.018 \\
\hline
\end{tabular}

NIHSS National Institutes of Health Stroke Scale, SE standard error, $\mathrm{Cl}$ confidence interval, OR odds ratio

[0 $(\leq-1), 1(>-1)]$, change in circumrotating during the first 3 days [0 (disappear); 2 (keep); 4 (appear)], change in tinnitus during the first 3 days [0 (disappear); 2.5 (keep); 5 (appear)]. The total score was calculated by adding up all factor scores. The maximum score was 16.5, and the range of score was from 0 to 16.5 points (Table 6).

Then a new 8-item scale for the early prediction of ischemic stroke recovery was developed to be used for clinical assessment. It means that the older patients, with a history of DM, high NIHSS score, anxiety, irritability on the 1st day, and with an increased NIHSS score, appearance of circumrotating and tinnitus during the first 3 days, were inclined to get poor recovery.

\section{Selection of cutoff value}

Receiver operating characteristic (ROC) curve analysis was performed to determine the cutoff value for the prognostic model of ischemic stroke recovery by using these 8 independent factors. The new scale was applied to estimate the prognosis of 413 ischemic stroke patients in 3 TCM hospitals by ROC curve analysis. And with prognostic assessment of ischemic stroke recovery measured by $\mathrm{BI}$ as the standard, the ROC curve indicated that at the cutoff point of 9.5 score, it had the greatest prognostic ability (maximum sensitivity and specificity) (Fig. 3).
The results of 310 cases $(\operatorname{good}=159$, poor $=151)$ in the $3 \mathrm{TCM}$ hospitals were predicted correctly, while the other 103 cases ( $\operatorname{good}=30$, poor $=73$ ) were wrong classified. The sensitivity of this model for prediction of good recovery was $68.5 \%$ and the specificity was $83.4 \%$. The overall probability of accurate prediction was $75.1 \%$. Therefore, the score of 9.5 was chosen as the cutoff value for prediction good and poor stroke recovery (Table 7).

\section{Validation of the scale for patients in Xuanwu hospital}

The validation result of the new scale showed that 57 cases $(\operatorname{good}=32$, poor $=25)$ were predicted correctly, while the other 19 cases $(\operatorname{good}=5$, poor $=14$ ) were wrong classified in Xuanwu hospital. The sensitivity and specificity of the scale for prediction of good recovery were 69.6 and $83.3 \%$, and the overall percentage of correct prediction was $75.0 \%$ (Table 7 ).

\section{Discussion}

\section{Summary of main findings}

The study showed that NIHSS score had a relationship with some TCM symptoms \& signs $(P<0.05)$, such as limb flaccid paralysis, anxiety, dizziness and so on. NIHSS is one of the most common assessment methods to predict the outcome of ischemic stroke. It found that

Table 6 A 8-item scale for the prediction of stroke recovery

\begin{tabular}{lll}
\hline Item & Weight & Assigned points \\
\hline Age & 1 & $0(\leq 56.44) ; 1(>56.44)$ \\
History of DM & 1 & 0 (no); 1 (yes) \\
Anxiety, day 1 & 1 & 0 (no); 1 (yes) \\
NIHSS score degree, day 1 & 1 & $0(\leq 2), 1(3-6), 2(>7)$ \\
Irritability, day 1 & 1.5 & 0 (no); 1.5 (yes) \\
Change in NIHSS score during 3 days & 1 & $0(\leq-1), 1(>-1)$ \\
Change in circumrotating during 3 days & 2 & 0 (disappear); 2 (keep); 4 (appear) \\
Change in tinnitus during 3 days & 2.5 & 0 (disappear); 2.5 (keep); 5 (appear) \\
\hline
\end{tabular}

NIHSS National Institutes of Health Stroke Scale 


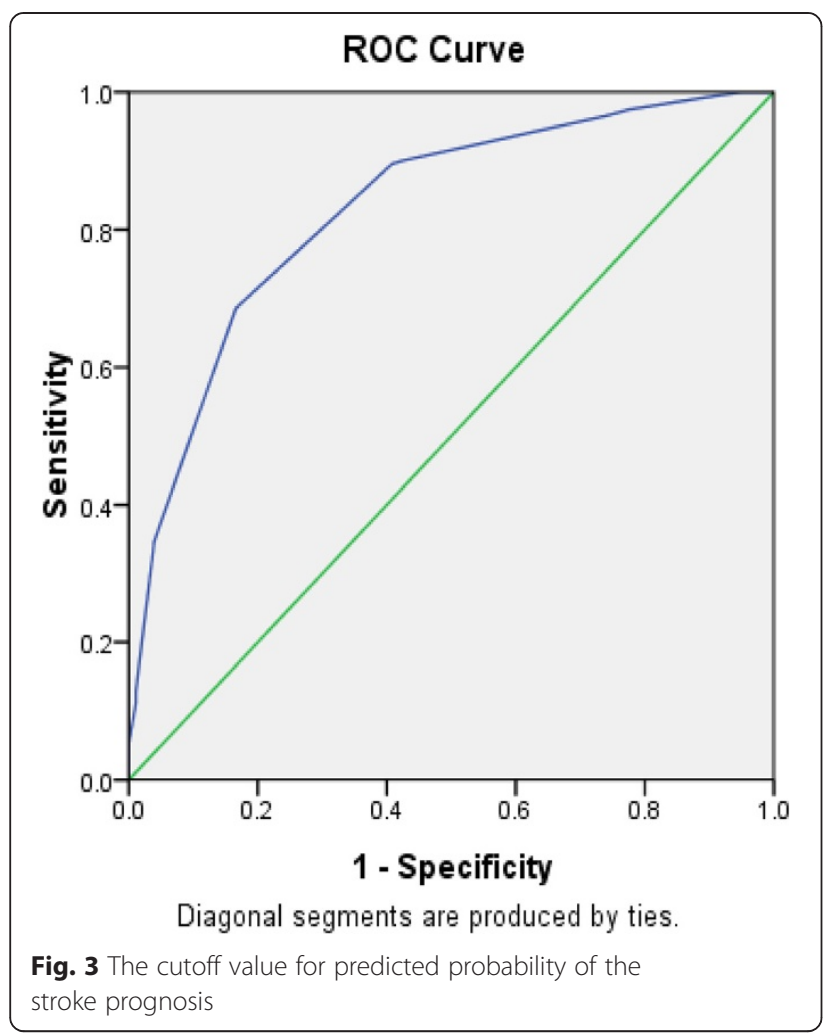

there was some relationship between NIHSS and certain TCM symptoms which indicates TCM symptoms may have a predictive value for the outcome of ischemic stroke. Many studies have shown that age is an independent factor in predicting patients' outcome [38, 39]. Consistent with previous researches, the result showed that patients aged 56.44 years old or younger were more likely to recover than those over 56.44 years old $(\mathrm{OR}=3.32$, $95 \%$ CI 1.72-6.42). Several studies have reported that NIHSS score had a significant effect on ischemic stroke outcome $[16,17]$. The study showed that the scale could make a more accurate prognosis using the change in NIHSS scores. The patients with an increased NIHSS score more than 1 during the first 3 days were

Table 7 An 8-item scale for the prediction of stroke recovery in TCM hospitals and Xuanwu hospital

\begin{tabular}{llllll}
\hline Hospital & $\begin{array}{l}\text { Good, } n \\
\text { TG }\end{array}$ & $\begin{array}{l}\text { Poor, } n \\
\text { FP }\end{array}$ & $\begin{array}{l}\text { Sensitivity } \\
(\%)\end{array}$ & $\begin{array}{l}\text { Specificity } \\
(\%)\end{array}$ & $\begin{array}{l}\text { Accuracy } \\
(\%)\end{array}$ \\
& FG & TP & & & \\
\hline 3TCM Hospitals & 159 & 73 & 68.5 & 83.4 & 75.1 \\
& 30 & 151 & & & \\
XW Hospital & 32 & 14 & 69.6 & 83.3 & 75.0 \\
& 5 & 25 & & & \\
\hline
\end{tabular}

$T G$ ture good recovery, $F G$ false good recovery, $T P$ ture poor recovery, $F P$ false poor recovery

Sensitivity $=T G /(T G+F P)$; Specificity $=T P /(T P+F G)$;

Accuracy $=(T G+T P) /(T G+F G+T P+F P)$
2.49 times $(\mathrm{OR}=2.49,95 \% \mathrm{CI} 1.31-4.73)$ more likely to get poor recovery. Some TCM symptoms and signs play an important role in prognosis. For example, the patients with anxiety on the 1st day were more likely to get poor recovery than those without it (OR $=3.17,95 \%$ CI 1.905.29). The disappearance of some TCM symptoms \& signs such as circumrotating and tinnitus during the first 3 days can also indicate good recovery.

Finally the new scale was composed of age degree, history of DM, NIHSS score, anxiety and irritability on the 1st day, and change in NIHSS score, circumrotating and tinnitus during the first 3 days (Table 8). The total score of the scale was 16.5 and the cutoff point was 9.5 by the ROC curve, which means patients would have poor recovery outcome on the 90th day if the score $>9.5$. The new scale was validated on Xuanwu hospital patients, and the sensitivity of the scale for the prediction of good recovery was $69.6 \%$ and the specificity was $83.3 \%$ with an overall accuracy $75.0 \%$.

\section{Strengths of the study}

To construct the scale by the TCM symptoms and NHISS

The four diagnostic methods in TCM include observation, listening and smelling, inquiry and pulse feeling diagnosis. These methods are not only the principal methods for collecting case information comprehensively, but also an important evidence for TCM differential syndrome and treatment, as well as a solid foundation for TCM clinical research and TCM clinical application [40]. Upholding TCM holism concepts as our guide, it can be of great significance to test the prognosis and recurrence of disease and guide the clinical practice by use of the comprehensive information. NIHSS scores can be used to measure the severity of stroke, which is highly predictive to excellent or devastating outcomes in ischemic stroke patients $[17,41]$. It also emphatically reflects the disease outcome at objective injury level, which has been considered a close association with prognosis [42]. The study combined the TCM diagnostic information with the evaluation tool of dysfunction in modern medicine to develop the scale, so as to be both comprehensive and focused. The 8-item scale for stroke outcome can comprehensively evaluate the prognosis from physical and psychological aspects without high cost. The study would provide a relatively comprehensive evaluation tool for clinical practice.

In addition, one previous study has shown that the prognostic model based on age and NIHSS score has correctly predicted survival and functional recovery after 3 months, which provided the basis for our research [11]. The modified Rankin Scale (mRS) is the most comprehensive and most widely used scale for measuring clinical outcome of stroke patients. However, substantial inter observer variability in $\mathrm{mRS}$ scoring has been 
Table 8 The new prognosis scale for the prediction of ischemic stroke recovery

\begin{tabular}{|c|c|c|c|}
\hline Item & Weight & Classify & Assigned point \\
\hline Age degree & 1.0 & & \\
\hline$\leq 56.44$ & & 0 & 0 \\
\hline$>56.44$ & & 1 & 1.0 \\
\hline History of Diabetes & 1.0 & & \\
\hline No & & 0 & 0 \\
\hline Yes & & 1 & 1.0 \\
\hline Anxiety, day 1 & 1.0 & & \\
\hline No & & 0 & 0 \\
\hline Yes & & 1 & 1.0 \\
\hline NIHSS score, day 1 & 1.0 & & \\
\hline$\leq 2$ & & 0 & 0 \\
\hline $2<$ NIHSS score $\leq 6$ & & 1 & 1.0 \\
\hline$>6$ & & 2 & 2.0 \\
\hline Irritability, day 1 & 1.5 & & \\
\hline No & & 0 & 0 \\
\hline Yes & & 1 & 1.5 \\
\hline NIHSS score change during 3 days & 1.0 & & \\
\hline$\leq-1$ & & 0 & 0 \\
\hline$>-1$ & & 1 & 1.0 \\
\hline Change in circumrotating during 3 days & 1.0 & & \\
\hline Disappear & & 0 & 0 \\
\hline Keep & & 1 & 2.0 \\
\hline Appear & & 2 & 4.0 \\
\hline Change in tinnitus during 3 days & 2.5 & & \\
\hline Disappear & & 0 & 0 \\
\hline Keep & & 1 & 2.5 \\
\hline Appear & & 2 & 5.0 \\
\hline Total score (sum of assigned points) ${ }^{a}$ & & & 16.5 \\
\hline
\end{tabular}

${ }^{\mathrm{a}}$ The range of score from 0 to 16.5 points; the cutoff point was 9.5

reported in a recent study, and the Rankin Focused Assessment (RFA) is suggested to be used in clinical trials as a short and practicable structured assessment tool [43]. Barthel index (BI) is a kind of activities of daily living (ADL) assessment tool with high reliability and sensitivity, commonly used in rehabilitation institutions of the United States. So BI was chosen as the prognostic assessment index in this study.

\section{The scale reflects the dynamic change in prognosis of ischemic stroke}

Stroke is a kind of disease with the characteristics of obvious stage change of symptoms and syndrome (acute phase/recovery/sequel period), which is manifested by acute onset and quick change [42]. The wind, fire, phlegm, blood stasis, and deficiency are the main syndrome factors of stroke. While NIHSS is mainly used for evaluating the degree of nerve function defect of acute ischemic stroke patients [17, 41], TCM symptoms and NIHSS reflect the development of stroke from different angles. Therefore, correlation researches on dynamic evolution of stroke syndrome elements and neurological function deficit have been conducted [44, 45]. Some researchers believe that there are certain and complex nonlinear relations between the two, while others have found they have correlations among them by use of the analysis method of Bayesian network, and the related degree would dynamic change over time [42]. Moreover, the result of the study was identical with the above conclusions. As a result, the new scale conforms to the characteristics of the dynamic development of the disease, and would more accurately evaluate the prognosis of stroke. 


\section{Limitation of the study}

The study has three limitations. Firstly, the patients are relatively milder in ordinary ward because the patients died or with incomplete record without BI data on the 90th day are not included in our study. Secondly, based on the second data analysis, the new scale needs to be further tested by prospective and lager sample size studies. Thirdly, further sensitivity tests and specificity by expert questionnaire and clinical verification is required.

\section{Conclusions}

The 8-item scale can be used to predict the 90-day outcome for ischemic stroke patients shortly after admission to hospital, but further clinical verification researches should be conducted to make the prediction rule more dependable for clinical use.

\section{Additional file}

Additional file 1: Table S1. TCM symptoms \& signs with appearance rate no less than $5 \%$. In practical analysis we selected 57 TCM symptoms with the appearance rate $\geq 5 \%$ from 157 TCM symptoms\& signs except tongue and pulse. (CSV $1 \mathrm{~kb}$ )

\section{Competing interests}

The authors declare that there are no competing interests.

\section{Authors' contributions}

All the authors participated in the preparation of the manuscript. KGC conceived the idea, designed the study and performed the statistical analysis. CHF has been involved in drafting the manuscript and revising it for important intellectual content. HQL participated in data acquisition and extraction, and helped to draft the manuscript. XYX helped with statistical analysis and interpretation of data. YG conceived the study and helped to ensure the accuracy and integrity of any part of the study. All authors read and approved the final manuscript

\section{Acknowledgements}

This study was supported by the Major State Basic Research Development Program of China (973 Program NO. 2003CB517102). The work was partially supported by National Key Technology R\&D Program during the"Twelfth Five-year"Plan (No.2012BAl25B01) and the Innovative Team Project of Beijing University of Traditional Chinese Medicine (No.2011-CXTD-22). The authors are grateful to professor Dimple Rajgor, Jatin Shah, and Ricardo Pietrobon of Duke-National University of Singapore Graduate Medical School for helpful statistical analysis.

\section{Author details}

${ }^{1}$ Department of Neurology, Dongzhimen Hospital affiliated to Beijing University of Chinese Medicine, Beijing, China. ${ }^{2}$ Department of Acupuncture and Moxibustion, Beijing Hospital of Traditional Chinese Medicine affiliated to Capital Medical University, Beijing, China. ${ }^{3}$ Traditional Chinese Medicine Department, Peking University Third Hospital, Beijing, China.

Received: 28 February 2015 Accepted: 7 October 2015

Published online: 16 November 2015

\section{References}

1. Lee JS, Hong JM, Moon GJ, Lee PH, Ahn YH, Bang OY, et al. A long-term follow-up study of intravenous autologous mesenchymal stem cell transplantation in patients with ischemic stroke. Stem Cells. 2010;28:1099-106.

2. $\mathrm{Yu}$ CY, Ng G, Liao P. Therapeutic antibodies in stroke. Transl Stroke Res. 2013:4:477-83
3. Rothwell PM, Coull AJ, Silver LE, Fairhead JF, Giles MF, Lovelock CE, et al. Population-based study of event-rate, incidence, case fatality, and mortality for all acute vascular events in all arterial territories (Oxford Vascular Study). Lancet. 2005;366:1773-83.

4. Liu HL, Zhang DS, Tan XG, Yang DQ, Wang GL, Zhao Y, et al. The effect of acupuncture on stroke recovery: study protocol for a randomized controlled trial. BMC Complement Altern Med. 2012;12:216.

5. Go AS, Mozaffarian D, Roger VL, Benjamin EJ, Berry JD, Blaha MJ, et al. Heart disease and stroke statistics-2014 update: a report from the American Heart Association. Circulation. 2014;129:e28-292.

6. Suljic E, Mehicevic A, Gavranovic A. Stroke emergency medical care: initial assessment, risk factors, triage and hospitalization outcome. Mater Sociomed. 2013;25:83-7.

7. Johnston SC, Mendis S, Mathers CD. Global variation in stroke burden and mortality: estimates from monitoring, surveillance, and modeling. Lancet Neurol. 2009;8:345-54.

8. Kim HJ, Kim SK, Park HJ, Chung JH, Chun J, Yun DH, et al. Polymorphisms of IGFI contribute to the development of ischemic stroke. Exp Ther Med. 2012;3:93-8.

9. Park $\mathrm{JH}$, Ovbiagele B. Optimal combination treatment and vascular outcomes in recent ischemic stroke patients by premorbid risk level. J Neurol Sci. 2015;355:90-3.

10. Larsson SC, Åkesson A, Wolk A. Primary prevention of stroke by a healthy lifestyle in a high-risk group. Neurology. 2015;84:2224-8.

11. König IR, Ziegler A, Bluhmki E, Hacke W, Bath PM, Sacco RL, et al. Predicting long-term outcome after acute ischemic stroke: a simple index works in patients from controlled clinical trials. Stroke. 2008;39:1821-6.

12. Hand PJ, Wardlaw JM, Rivers CS, Armitage PA, Bastin ME, Lindley Rl, et al. MR diffusion-weighted imaging and outcome prediction after ischemic stroke. Neurology. 2006;66:1159-63.

13. Park $\mathrm{CH}$, Chang $\mathrm{WH}$, Ohn SH, Kim ST, Bang OY, Pascual-Leone A, et al. Longitudinal changes of resting-state functional connectivity during motor recovery after stroke. Stroke. 2011;42:1357-62.

14. Sare GM, Ali M, Shuaib A, Bath PM, VISTA Collaboration. Relationship between hyperacute blood pressure and outcome after ischemic stroke: data from the VISTA collaboration. Stroke. 2009:40:2098-103.

15. Heldner MR, Zubler C, Mattle HP, Schroth G, Weck A, Mono ML, et al. National Institutes of Health stroke scale score and vessel occlusion in 2152 patients with acute ischemic stroke. Stroke. 2013;44:1153-7.

16. Kasner SE, Chalela JA, Luciano JM, Cucchiara BL, Raps EC, McGarvey ML, et al. Reliability and validity of estimating the NIH Stroke Scale score from medical records. Stroke. 1999:30:1534-7.

17. Boone M, Chillon JM, Garcia PY, Canaple S, Lamy C, Godefroy O, et al. NIHSS and acute complications after anterior and posterior circulation strokes. Ther Clin Risk Manag. 2012;8:87-93.

18. Ghandehari K, Ghandehari K, Saffarian-Toosi G, Masoudinezhad S, Yazdani S, Nooraddin A, et al. Comparative interrater reliability of Asian Stroke Disability Scale, modified Rankin Scale and Barthel Index in patients with brain infarction. ARYA Atheroscler. 2012:8:153-7.

19. Dionyssiotis Y, Kiourtidis D, Karvouni A, Kaliontzoglou A, Kliafas I. Consequences of neurologic lesions assessed by Barthel Index after Botox (๑) injection may be underestimated. Ther Clin Risk Manag. 2012;8:385-91.

20. Djelilovic-Vranic J, Alajbegovic A, Zelija-Asimi V, Niksic M, Tiric-Campara M, Salcic $S$, et al. Predilection role diabetes mellitus and dyslipidemia in the onset of ischemic stroke. Med Arh. 2013;67:120-3.

21. Meschia JF, Bushnell C, Boden-Albala B, Braun LT, Bravata DM, American Heart Association Stroke Council; Council on Cardiovascular and Stroke Nursing; Councilon Clinical Cardiology; Council on Functional Genomics and Translational Biology; Council on Hypertension, et al. Guidelines for the primary prevention of stroke: a statement for healthcare professionals from the American Heart Association/ American Stroke Association. Stroke. 2014:45:3754-832.

22. Liu GP, Li GZ, Wang YL, Wang YQ. Modelling of inquiry diagnosis for coronary heart disease in traditional Chinese medicine by using multi-labe learning. BMC Complement Altern Med. 2010;10:37.

23. Li GZ, Sun S, You MY, Wang YL, Liu GP. Inquiry diagnosis of coronary heart disease in Chinese medicine based on symptom-syndrome interactions. Chin Med. 2012;7:9.

24. Li GZ, Yan SX, You MY, Sun S, Ou AH. Intelligent ZHENG classification of hypertension depending on ML-kNN and feature fusion. Evid Based Complement Alternat Med. 2012;2012:837245. 
25. Hua T. Zhong Zang Jing. Hua T. Zhong Zang Jing. Jiangsu Science and Technology Press 1985:14-16.

26. Gao Y, Zhong HZ, Ma B, Huang Y. Randomized, double-blind, parallelcontrolled clinical trial on correspondence of prescriptions and traditional Chinese medicine syndrome of ischemic stroke. J Tradit Chin Med. 2012;32:335-42

27. Liu L, Gao Y. Study on the correlation between traditional Chinese medicine syndrome and short-term prognosis of ischemic stroke using logistic regression model and repeated-measures analysis of variance. Zhong Xi Yi Jie He Xue Bao. 2012;10:983-90.

28. Gialanella B, Santoro R, Ferlucci C. Predicting outcome after stroke: the role of basic activities of daily living predicting outcome after stroke. Eur J Phys Rehabil Med. 2013:49:629-37.

29. World Medical Association. World Medical Association Declaration of Helsinki: ethical principles for medical research involving human subjects. JAMA. 2013;310:2191-4.

30. Stroke-1989. Recommendations on stroke prevention, diagnosis, and therapy. Report of the WHO task force on stroke and other cerebrovascular disorders. Stroke. 1989;20:1407-31.

31. The Encephalopathy Accident and Emergency Collaborative Group of State Administration of Traditional Chinese Medicine. Criteria of diagnosis and therapeutic effect evaluation for apoplexy (trial version). Beijing Zhong Yi Yao Da Xue Xue Bao. 1996;19:55-6.

32. Cioncoloni D, Piu P, Tassi R, Acampa M, Guideri F, Taddei S, et al. Relationship between the modified Rankin scale and the Barthel index in the process of functional recovery after stroke. Neuro Rehabilitation. 2012;30:315-22.

33. Mahoney FI, Barthel DW. Functional evaluation: the Barthel Index. Md State Med J. 1965;14:61-5.

34. Houlden H, Edwards M, McNeil J, Greenwood R. Use of the Barthel index and the functional independence measure during early inpatient rehabilitation after single incident brain injury. Clin Rehabil. 2006;20:153-9.

35. Kauranen T, Laari S, Turunen K, Melkas M, Mustanoja S, Baumann P, et al. Use of stroke-related income supplements and predictors of Use in a working-aged Finnish ischemic stroke cohort. J Stroke Cerebrovasc Dis. 2015;24:1715-23.

36. Prasad K, Dash D, Kumar A. Validation of the Hindi version of National Institute of Health Stroke Scale. Neurol India. 2012;60:40-4.

37. Middleton S, Grimley R, Alexandrov AW. Triage, treatment, and transfer: evidence-based clinical practice recommendations and models of nursing care for the first $72 \mathrm{~h}$ of admission to hospital for acute stroke. Stroke. 2015;46:e18-25.

38. Lin PH, Yeh WT, Svetkey LP, Chuang SY, Chang YC, Wang C, et al. Dietary intakes consistent with the DASH dietary pattern reduce blood pressure increase with age and risk for stroke in a Chinese population. Asia Pac J Clin Nutr. 2013;22:482-91

39. Hanson E, Nilsson S, Jood K, Norrving B, Engström G, Blomstrand C, et al Genetic variants of coagulation factor XI show association with ischemic stroke Up to 70 years of Age. PLoS One. 2013;8:e75286.

40. Shi Q, Zhao H, Chen J, Ma X, Yang Y, Zheng C, et al. Study on TCM syndrome identification modes of coronary heart disease based on data mining. Evid Based Complement Alternat Med. 2012;2012:697028. 10.1155 /2012/697028

41. Tan F, Wang X, Li HQ, Lu L, Li M, Li JH, et al. A randomized controlled pilot study of the triple stimulation technique in the assessment of electroacupuncture for motor function recovery in patients with acute ischemic stroke. Evid Based Complement Alternat Med. 2013;2013:431986.

42. Jiang LJ, Hu JQ, Yi DH, Chen Q, Liu BY. Bay esian network analysis of correlation between TCM syndrome dynamic changes and NIHSS scores of ischemia stroke. World Chinese Medicine. 2013;8:613-7.

43. Saver JL, Filip B, Hamilton S, Yanes A, Craig S, Cho M, et al. Improving the reliability of stroke disability grading in clinical trials and clinical practice: the Rankin Focused Assessment (RFA). Stroke. 2010;41:992-5.

44. Zhang H, Gao Y. Correlation between syndrome changes and neurological parafunction of ischemia stroke. J Beijing University Traditional Chin Med. 2007;30:274-8

45. Wang ZH, Liu C, Xu JF, Li XY, Zhang YX, Gao Y, et al. Study of relationship of NIHSS and TCM standardized sheet of apoplexy syndromes diagnosis base on LVQ neural networks. Liaoning J TCM. 2009;36:2020-2.

\section{Submit your next manuscript to BioMed Central and take full advantage of:}

- Convenient online submission

- Thorough peer review

- No space constraints or color figure charges

- Immediate publication on acceptance

- Inclusion in PubMed, CAS, Scopus and Google Scholar

- Research which is freely available for redistribution

Submit your manuscript at www.biomedcentral.com/submit 